ORIGINAL ARTICLE

\title{
Detection of Nocardia, Streptomyces and Rhodococcus from broncho- alveolar lavage specimens of patients with HIV by Multiplex PCR Assay
}

\author{
Hossein Ali Rahdar, ${ }^{1,2}$ Mohammad Reza Salehi ${ }^{3}$, Abass Bahador ${ }^{1}$, Seyedesomaye \\ Jasemi $^{1}$, Morteza Karami-Zarandi ${ }^{1}$, Malehe Hasan Nejad $^{3}$, Shahram Shahraki- \\ Zahedani $^{4}$, Jafar Amani ${ }^{5}$, Seifu Gizaw Feyisa ${ }^{1,6}$, Jalil Kardan-Yamchi ${ }^{1}$, Mohammad \\ Mehdi Feizabadi $^{{ }^{*}}$
}

\footnotetext{
OPEN ACCESS

Citation: Hossein Ali Rahdar, Mohammad Reza Salehi, Abass Bahador, et al. Detection of Nocardia, Streptomyces and Rhodococcus from broncho-alveolar lavage specimens of patients with HIV by multiplex PCR assay. Ethiop J Health Sci. 2019; 29(6):737.doi:http://dx.doi.org/10.4314/ej hs.v29 i6.10

Received: June 28, 2019

Accepted: July 27, 2019

Published: November 1, 2019

Copyright: (C2019 Hossein Ali Rahdar, et al. This is an open access article distributed under the terms of the Creative Commons Attribution License, which permits unrestricted use, distribution, and reproduction in any medium, provided the original author and source are credited.

Funding: This study was supported by Tehran University of Medical Sciences (Project No,36924).

Competing Interests: The authors declare that this manuscript was approved by all authors in its form and that no competing interest exists.

Affiliation and Correspondence:

${ }^{1}$ Department of Medical Microbiology,

School of Medicine, Tehran University of

Medical Sciences, Tehran, Iran.

${ }^{2}$ Department of Microbiology, School of

Medicine, Iranshahr University of Medical

Sciences, Iranshahr, Iran

${ }^{3}$ Department of Infectious and Tropical

Diseases, Imam Khomeini Hospital

Complex, Tehran University of Medical

Sciences, Tehran, Iran.

${ }^{4}$ Department of Medical Microbiology,

School of Medicine, Zahedan University of

Medical Sciences, Zahedan, Iran.

${ }^{5}$ Baqiyatallah University of Medical

Sciences, Tehran, Iran.

${ }^{6}$ Department of Biology, College of Natural

Sciences, Jimma University, Ethiopia

*Email: mfeizabadi@tums.ac.ir
}

\begin{abstract}
Background: Nocardia, Streptomyces and Rhodococcus are life threatening opportunistic pathogens under immunodeficiency conditions, particularly among patients infected with HIV. Rapid and accurate detection of these infections can improve immune health quality, patient management and appropriate treatment. The aim of this study was to design a novel multiplex-PCR assay for rapid diagnosis of these three organisms directly from bronchoalveolar lavage (BAL) specimens of patients infected with HIV.

Methods: The genus specific primers were designed for directdetection of Nocardia, Streptomyces and Rhodococcus in a single tube multiplex PCR. This PCR specifically amplified the target genes from pure cultures. It subsequently was applied on $B A L$ specimens of $29 \mathrm{HIV}$ positive patients that had previously been culture negative for actinomycete bacteria, of which Nocardia, Streptomyces and Rhodococcus are members.

Results: Of 29 respiratory clinical specimens, there were positive for Nocardia spp. and one was positive for Streptomyces spp using the multiplex PCR assay. The sequencing of the PCR products identified the species as Nocardia cyriacigeorgica $(n=2)$, Nocardia farcinica and Streptomyces albus.

Conclusion: This novel multiplex PCR assay yielded reliable results for accurate identification of Nocardia, Streptomyces and Rhodococcus from BAL while the results of bacterial culture were negative.

Keywords: Nocardia; Streptomyces; Rhodococcus; HIV; Infection; Multiplex PCR.
\end{abstract}

\section{INTRODUCTION}

In recent years, the incidence of opportunistic infections has increased in individuals with underlying conditions, autoimmune diseases, cancers, HIV, organ transplantation and chemotherapy. Among opportunistic pathogens, members of actinomycetes order such as Nocardia, Streptomyces and Rhodococcus need to be identified correctly prior to starting antibiotic therapy $(1,2)$. 
Pulmonary nocardiosis is an invasive infection caused by the genus Nocardia, a partial acid-fast microorganism. The common route of pulmonary infection is inhalation of contaminated aerosols from environmental sources. Therefore, the lung is the most common organ involved in nocardiosis. Other entry routes include direct bacterial inoculation of the skin and eyes $(1,3)$. Nocardia species may be transmitted to immunocompromised individuals through contact with environmental sources, leading to various complications, from local cutaneous lesions to disseminated infections (3).

Streptomyces spp. are gram-positive filamentous bacteria that live in a wide range of environmental sources and can produce antimicrobial compounds and secondary metabolites. This group of bacteria may cause opportunistic infections such as pulmonary disease, tuberculosis mimicking, and mycetoma in immunocompromised patients (4).

Rhodococcus spp. are saprophytic members of Nocardiaceae that live in environmental sources including soil, dust, decaying plants, milk and animals. The first case of infection with Rhodococcus equi was reported in 1967 by Golub et al. from lung abscess of a patient with AIDS $(5,6)$. In addition, there are several clinical reports of human infections with $R$. erythropolis, $R$. ruber, $R$. gordoniae, and $R$. fascians. However, $R$. equi has been the predominant species causing infections among rhodococci $(5,7)$. Nowadays, the incidence rate of infections with Rhodococcus species are increasing due to an increasing number of individuals with immune-disorders and the development of microbiology methods to detect these bacteria $(5,8,9)$.

We are at the end of the third decade of the acquired AIDS pandemic (10). According to the literature, the number of opportunistic infections, particularly aerobic-actinomyces infection in HIVinfected patients has increased (11). Although, culture is a gold standard method for laboratory diagnosis of bacterial diseases, this method is not robust because of the slow growing of the actinomycete bacteria and complexity of their nutritional requirement (12). In addition, it cannot differentiate between clinical symptoms of pneumonia; skin, sub-cutaneous and brain abscesses caused by actinomycetes from tuberculosis, viral, fungi infection or malignant tumours (3). Moreover, the sensitivity of direct smear microscopy and culture is low and controversial as it interferes with prior use of antibiotics before sampling $(1,13)$. These methods are also time consuming, expensive and need technical skill. Therefore, application of rapid, inexpensive and reliable diagnostic methods is essential for diagnosis and management of these infections. Previously, PCR was used in another study for the diagnosis of these infections in separate single tubes $(14,15)$. The aim of this study was to design a novel multiplex-PCR assay for simultaneous identification of Nocardia and Streptomyces and Rhodococcus genera infections from broncho-alveolar lavage (BAL) specimens of $\mathrm{HIV}$-infected patients in a single tube.

\section{MATERIALS AND METHODS}

Culturing of specimens: This study was performed between November 2018 and March 2019 at the Imam Khomeini Complex Hospital (IKH), Tehran University of Medical Sciences (Tehran, Iran). Totally, 29 clinical respiratory specimens including one lung biopsy as well as 28 BAL specimens were collected during routine bronchoscopy from complicated pulmonary infections of HIV patients excluding pulmonarytuberculosis. The specimens were sent to the Department of Molecular Microbiology laboratory at Tehran University of Medical Sciences immediately and divided into two tubes.

The specimens in the first tube were cultured on brain-heart infusion (BHI) agar medium containing polymyxin B (5 mg/liter), vancomycin $(5 \mathrm{mg} / \mathrm{liter})$ and cycloheximide $(5 \mathrm{mg} / \mathrm{liter})$ and incubated for 2 to 4 weeks at 30 and $37^{\circ} \mathrm{C}$ (3). Direct smear was prepared on slides and specimens were stained with partially acid-fast and Gram staining using the specimens from the first tube. The specimens in the second tube were used for PCR assay.

This study has been approved by the ethical committee of Tehran University of Medical Sciences (approval number, IR.TUMS.MEDICINE.REC.1397.261).

Primer design: The gyrB gene sequences were retrieved from NCBI Gene Bank for the various

DOI: http://x.doi.org/10.4314/ejhs.v29i6.10 
genera including Dietzia, Gordonia, Mycobacterium, Nocardia, Rhodococcus, Streptomyces, and Tsukamurella to design genus specific primers pairs. Several gyrB gene sequences were obtained for every genus. The sequences were analysed by Oligo 5 Software and signature sequences for each genus were selected.

Table 1. Primers designed for Nocardia (NR), Streptomyces (Stp) and Rhodococcus equi (R).

\begin{tabular}{|c|c|c|c|}
\hline Primer & Sequence & Length of product & genus \\
\hline Stp-F & GTGCTGTGCCAGAAAGGGCG & \multirow[b]{2}{*}{$320 \mathrm{bp}$} & \multirow[b]{2}{*}{ Streptomyces spp } \\
\hline Stp-R & GCGAGGATCGTGACGTCGAT & & \\
\hline NR-F & CGACCACAAGGGGGCCTA & \multirow{2}{*}{$596 \mathrm{bp}$} & \multirow{2}{*}{ Nocardia spp. } \\
\hline NR-R & GGTTGTAAACCTCTTTCGACAGG & & \\
\hline R-F & TCCAGAAGCGGGATGAGGAT & \multirow{2}{*}{$707 \mathrm{bp}$} & \multirow{2}{*}{ Rhodococcus spp. } \\
\hline R-R & TGGTGTGATGGCGGAAGATC & & \\
\hline
\end{tabular}

Specificity of the PCR assay: Samples used:DNA from 33 clinical isolates of Nocardia species $(\mathrm{n}=10)$, Nocardiopsis species $(\mathrm{n}=2)$, Streptomyces species $(\mathrm{n}=2)$, Rhodococcus equi $(\mathrm{n}=1)$, different species of non-tuberculosis mycobacteria $(\mathrm{n}=4)$, Mycobacterium tuberculosis $s(\mathrm{n}=2)$, Klebsiella pneumoniae $(\mathrm{n}=3)$, Acinetobacter baumannii strains $(\mathrm{n}=3)$, Pseudomonas aeruginosa $(\mathrm{n}=3)$, Staphylococcus aureus $(\mathrm{n}=3), \quad$ Nocardia cyriacigeorgica (DSM44484), N. asteroids (ATCC19247) and N. otitidiscaviarum (ATCC14629) were tested to determine the specify of the multiplex PCR.

DNA extraction: Chromosomal DNA was extracted using the simple boiling method. In brief, several colonies of bacteria were added to $200 \mathrm{ml}$ TE buffer. Subsequently, the suspension was boiled for $30 \mathrm{~min}$ and centrifuged at $11,000 \mathrm{~g}$ for $10 \mathrm{~min}$. The supernatant was transferred to another sterile micro tube and centrifuged at $20,000 \mathrm{~g}$ for $10 \mathrm{~min}$. The DNA-pellet was resuspended in $50 \mu 1$ Milli-Q water and stored at -20 ${ }^{\circ} \mathrm{C}$ (3) for molecular assay.

PCR amplification: PCR was carried out in reaction mixture containing $12.5 \mu \mathrm{L}$ master mix
A pair of primers was designed for pathogenic genera of Streptomyces. By modifications in existing primers, pairs of specific primers were designed for Nocardia and Rhodococcus equi (Table 1) $(16,17)$. 
(7). In order to extract DNA from the second tube of BAL specimen, $100 \mu \mathrm{L}$ of BAL samples was suspended in $250 \mu \mathrm{L}$ of sterile distilled water and then DNA was extracted using MTB respiratory specimen preparation kit (Invitek, USA).

Multiplex PCR was performed as above using specific primers for the genera of Nocardia, Streptomyces and Rhodococcus equi for direct identification of these pathogens from BAL samples. Positive results from the gel were confirmed by DNA sequencing. Sequences were compared with those stored in GenBank using BLAST alignment software. Similarity greater than $99 \%$ was considered as a single species.

Clinical data collection: Patient's demographic data such as age, genus, BAL leukocyte count, cotrimoxazole consumption, C-reactive protein, alveolar cavitation and other clinical signs were collected, according to privacy and ethical principles. After sequencing, treatment choices in respect to the bacteria detected were documented, along with the follow-up of the patient (discussed in the Discussion section).

\section{RESULTS}

\section{Specificity testing of the method}

Study population of bacterial samples: In total, 28 BAL specimens and one lung biopsy from HIV-infected patients (18 men and 11 women; mean age: 41 years old) were collected by bronchoscopy for diagnosis of aerobic actinomycetes infection (particularly Nocardiosis) (Table 2). Twelve patients had started therapy by low dosage of co-trimoxazole prophylaxis before sampling of bronchoscopy. All specimens were negative in culture, although two specimens were positive in direct smear microscopy.

Table 2. Main characteristics of study population $(n=29)$. BAL, broncho-alveolar lavage. A detailed version of this table is available as Underlying data.

\begin{tabular}{lll}
\hline & $\begin{array}{l}\text { Multiplex-PCRBALs+lung } \\
\text { biopsy }\end{array}$ & $\begin{array}{l}\text { Multiplex-PCR Bative } \\
\text { lung biopsy positive }\end{array}$ \\
\hline Male & 15 & 3 \\
Female & 10 & 1 \\
Median age (years) & 41 & 41 \\
Cotrimoxazole prophylaxis (n) & 11 & 1 \\
Blood tests at time of BAL & & \\
Median white blood cell count $(\mathrm{x} 1000 / \mu \mathrm{L})$ & 6.2 & 7.1 \\
Median neutrophil count $(\mathrm{x} 1000 / \mu \mathrm{L})$ & 3.7 & 5.1 \\
Median lymphocyte count $(\mathrm{x} 1000 / \mu \mathrm{L}$ & 1.6 & 1.1 \\
Median CRP concentration $(\mathrm{mg} / \mathrm{L})$ & 5.1 & 3.2 \\
PCR result (n) & 25 & 4 \\
Clinical/radiological presentation $(n)$ & & 2 \\
Pulmonary cavity & 8 & 2 \\
Pulmonary abscess & 1 & 4 \\
Chronic bronchopulmonary & 6 & 1 \\
Brain abscess & 2 & \\
\hline
\end{tabular}

Multiplex PCR assay and sequencing: From 29 negative culture specimens, 4 showed positive results in multiplex PCR assay including 3 specimens that were positive for Nocardia and one for Streptomyces species. The sizes of PCR products were 596 bp (Nocardia spp.), 320bp (Streptomyces spp.) and 707 bp (Rhodococcus spp.) (Figure 1). 


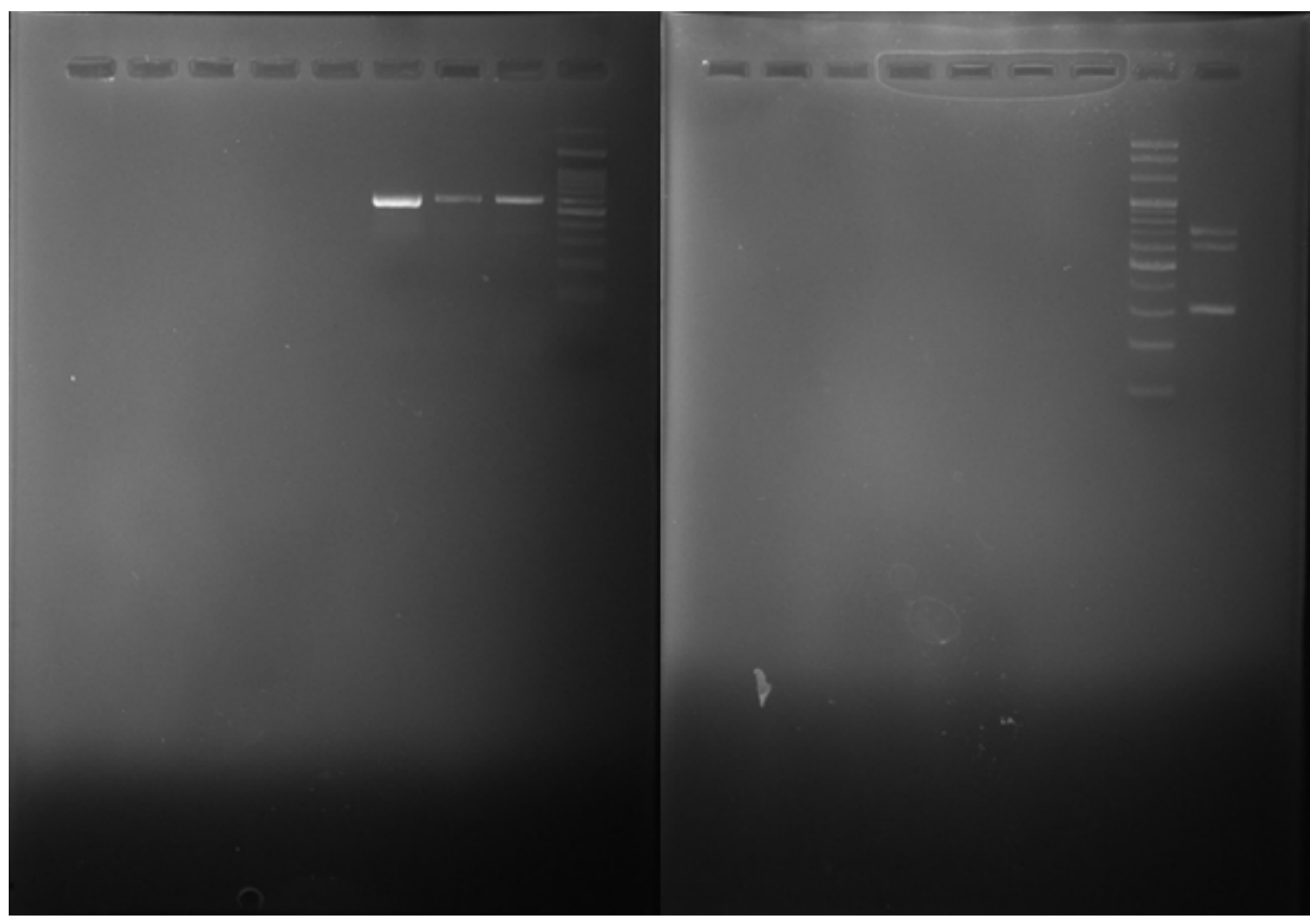

Figure 1 Multiplex-PCR - amplified products in 1\% agarose gel; Nocardia (596bp), Streptomyces (320 bp), Rhodococcus (707 bp), and M, molecular size markers (100-bp ladder).

Sequencing of products $(\mathrm{n}=4)$ identified the organisms as Nocardia cyriacigeorgica $(\mathrm{n}=2), N$. farcinica $(\mathrm{n}=1)$ and Streptomyces albus $(\mathrm{n}=1)$. Among PCR positive patients, 4 were suffering from chronic broncho-pneumonia and one patient had co-infection of Klebsiella pneumoniae and Nocardia cyriacigeorgica. The results of sequencing confirmed the accuracy of PCR assay (GeneBank accession numbers: MK968270, MK968271, MK968277, MN043685).

Characteristics of study population and multiplex PCR sensitivity assessment: From four PCR positive patients, two were positive in direct smear (partially acid-fast and Gram staining) and took co-trimoxazole before specimen collection. This may be the reason for negative result of culture in this patient. Three other patients did not use antibiotics prior to the specimen collection. All 4 patients were suffering from lung cavitation or lung abscess. One patient had disseminated nocardiosis, which is caused by Nocardia cyriacigeorgica. This organism was isolated from both brain abscess and BAL specimens.

\section{DISCUSSION}

This study was the first pilot study for directdetection of Nocardia, Streptomyces and Rhodococcus genera infections in respiratory clinical specimens using multiplex-PCR method. We detected three cases of infection with Nocardia spp. as well as one case of pulmonary infection by Streptomyces in HIV-infected patients. The results were confirmed by DNA sequencing. In addition, the sensitivity of this PCR for culture negative samples was demonstrated to be high; the results demonstrated that our primers have high efficiency for identification of Nocardia and Streptomyces from clinical specimens. It appears that this method is not inhibited by natural inhibitors within the specimens.

The slow growing rate of these organisms, antibiotic consumption before sample collection and time consuming phonotypical tests mean a 
delay in treatment, which increases the mortality rate among $\mathrm{HIV}$-infected cases $(18,19)$. Rapid identification in early stages of infection and selection of suitable antibiotics increase the success rate for treatment (20). Therefore, it is important to use molecular techniques as rapid and accurate methods for identification of these agents from clinical specimens to save the time and improve therapy.

In our study, three nocardiosis patients and one Streptomyces infected patients showed symptoms at presentation. One of the patients with nocardiosis, one had used co-trimoxazole before collecting the specimens. In clinical samples (especially cerebral and skin abscesses samples) growth of bacteria can be postponed or inhibited by pre-sampling antibiotic consumption. Molecular tests are independent of bacterial cell growth and could be remedial in correct diagnosis of bacteria. Also, one of the patients in this study had co-infection of Nocardia with Klebsiella pneumoniae. In previous studies, co-infection of Nocardia with other bacterial pathogens was reported in $10-30 \%$ of patients $(1,21-22)$. $N$. cyriacigeorgica appears to be the most frequent Nocardia species isolated from Iranian patients. It is intrinsically resistant to ciprofloxacin, one of the therapeutic choices for respiratory tract infections $(23,24)$. Early detection of $N$. cyriacigeorgica in clinical samples can lead physicians to choose the correct therapy. Brain abscesses is a lifethreatening infection and with $40-50 \%$ mortality (25). The result of culture for BAL was negative in a patient with brain abscess, while multiplex PCR detected the causative as $N$. cycriarogeorgica. In follow-up, after antinocardiosis treatment of co-trimoxazole, the brain abscess was diminished, indicating that multiplex PCR improved diagnosis and treatment.

In this study, $N$. farcinica strain was detected by multiplex-PCR method, while the culture was negative for the specimen of the same patient. High rate of resistance to antibiotics such as TMPSXT has been reported among $N$. farcinica isolates (26). Therefore, rapid detection of this species is very important in order to prevent treatment failure. In follow-up, after diagnosis of $N$. farcinica, the treatment was changed from TMP-SXT to imipenem and subsequently the respiratory signs of the patient improved. It has been suggested that recurrence of nocardiosis is common and in several studies it has been shown that recurrence is due to cell wall deficient bacterial cells, which may not be identifiable in culture (27-29). Moreover, Nocardia infections show similar signs to Parkinson's disease (30-31); therefore, molecular diagnostic methods can easily identifiy the infectious agent from this nervous system disease.

Pulmonary infection by Streptomyces has been increasing due to immune deficiency in recent years (32). The clinical signs of Streptomyces infection mimics tuberculosis (32), and as there are differences between the methods used for processing and culturing Streptomyces and Mycobacterium tuberculosis, misdiagnosis of these two infections may occur. This may be complicating treatment as the choices of treatment are also different. In our study S. albus infection case showed symptoms similar to tuberculosis (presence of granulomas) (32). Anti-tuberculosis regimen is not effective for treatment of infection with this bacterium. After getting the positive multiplex-PCR result for $S$. albus, treatment was started using imipenem. Therefore, multiplex-PCR was useful for the timely selection of the correct drug regimen and preventing treatment failure.

Although Rhodococcus equi was not detected in our study, it is very important in immune deficiency and people working with animals (9); one third of patients who are infected by $R$. equi have a history of contact with horses or pigs. Transmission has also been reported during handling and working with clinical samples (5). Therefore, rapid detection of this bacteria is needed in high risk patients and those in contact with contaminated sources.

Therefore, molecular methods can differentiate these agents that are completely different in laboratory detection and clinical treatment but very similar in clinical signs.

In conclusion, new emerging pathogens such as Nocardia, Streptomyces and Rhodococcus are being isolated from clinical samples more frequently in recent years due to increases in immune deficiency diseases like HIV infections. Improvement of diagnostic methods in screening and identification of these bacterial infections are

DOI: http://dx.doi.org/10.4314/ejhs.v29i6.10 
highly demanded. Nocardia genus has had more attention and been studied more, but Streptomyces and Rhodococcus species also are increasing. In this study, we designed a novel multiplex PCR for detection of infected cases with Nocardia, Streptomyces and Rhodococcus species for the first time. The results of our study are equivalent or may be better than conventional or current inhouse PCR methods. The high specificity $(85.7 \%)$ and sensitivity $(100 \%)$ of this method suggest that the development of this multiplex-PCR is a reliable diagnostic tool for direct detection of Nocardia, Streptomyces and Rhodococcus infections from BAL and possibly other clinical specimens.

\section{AKNOWLEDGMENTS}

The authors are grateful to Office of Vicechancellor for Research of Tehran University of Medical Sciences for the support of the current study (grant number: 36924).

\section{REFERENCES}

1. Brown-Elliott BA, Brown JM, Conville PS, Wallace RJ, Jr. 2006. Clinical and laboratory features of the Nocardiaspp. based on current molecular taxonomy.ClinMicrobiol Rev 19:259-282.

2. Coussement J, Lebeaux D, van Delden C, Guillot H, Freund R, Marbus S, Melica G, Van Wijngaerden E, Douvry B, Van Laecke S, Vuotto F, Tricot L, Fernández-Ruiz M, Dantal J, Hirzel C, Jais J, Rodriguez-Nava V, Lortholary O, Jacobs F. 2016. Nocardiainfection in solid organ transplant recipients: multicenter European case-control study. Clin Infect Dis 63:338 -345.

3. Rahdar HA, Azadi D, Shojaei H, Daei-Naser A. Molecular analysis and species diversity of Nocardia in the hospital environment in a developing country, a potential health hazard. Journal of medical microbiology. 2017 Mar 23;66(3):334-41.

4. Kapadia M, Rolston KV, Han XY. Invasive Streptomyces infections: six cases and literature review. American Journal of Clinical Pathology. 2007 Apr 1;127(4):61924.
5. Majidzadeh M, Fatahi-Bafghi M. Current taxonomy of Rhodococcus species and their role in infections. European Journal of Clinical Microbiology \& Infectious Diseases. 2018;37(11):2045-62.

6. Bell KS, Philp JC, Aw DW, Christofi N. The genus Rhodococcus. Journal of Applied Microbiology. 1998;85(2):195-210.

7. Witkowski L, Rzewuska M, Takai S, Kizerwetter-Świda M, Kita J. Molecular epidemiology of Rhodococcus equi in slaughtered swine, cattle and horses in Poland. BMC microbiology. 2016;16(1):98.

8. Kedlaya I, Ing MB, Wong SS. Rhodococcus equi infections in immunocompetent hosts: case report and review. Clinical infectious diseases. 2001;32(3):e39-46.

9. Weinstock DM, Brown AE. Rhodococcusequi: an emerging pathogen. Clinical Infectious Diseases. 2002 May 15;34(10):1379-85.

10. de Béthune MP. Non-nucleoside reverse transcriptase inhibitors (NNRTIs), their discovery, development, and use in the treatment of HIV-1 infection: a review of the last 20 years (1989-2009). Antiviral research. 2010 Jan 1;85(1):75-90.

11. Chaudhry SI, Greenspan JS. Actinomycosis in HIV infection: a review of a rare complication. International journal of STD \& AIDS. 2000 Jun 1;11(6):349-55.

12. Keikha M. Williamsia spp. are emerging opportunistic bacteria. New microbes and new infections. 2018;21:88-9.

13. Coussement J, Lebeaux D, Rouzaud C, Lortholary O. Nocardiainfections in solid organ and hematopoietic stem cell transplant recipients. CurrOpin Infect Dis 2017; 30(6): $545-51$.

14. Coussement J, Lebeaux D, El Bizri N, Claes V, Kohnen M, Steensels D, Étienne I, Salord $\mathrm{H}$, Bergeron E, Rodriguez-Nava V. Nocardia polymerase chain reaction (PCR)-based assay performed on bronchoalveolar lavage fluid after lung transplantation: A prospective pilot study. PloS one. 2019 Feb 25;14(2):e0211989.

15. Rouzaud C, Rodriguez-Nava V, Catherinot E, Méchaï F, Bergeron E, Farfour E, Scemla A,

DOI: http://dx.doi.org/10.4314/ejhs.v29i6.10 
Poirée S, Delavaud C, Mathieu D, Durupt S. Clinical assessment of a Nocardia PCR-based assay for diagnosis of nocardiosis. Journal of clinical microbiology. 2018 Jun 1;56(6):e00002-18.

16. Laurent FJ, Provost F, Boiron P. Rapid Identification of Clinically RelevantNocardia Species to Genus Level by $16 \mathrm{~S}$ rRNA Gene PCR. Journal of clinical microbiology. 1999 Jan 1;37(1):99-102.

17. Arriaga JM, Cohen ND, Derr JN, Chaffin MK, Martens RJ. Detection of Rhodococcus equi by polymerase chain reaction using speciesspecific nonproprietary primers. Journal of veterinary diagnostic investigation. 2002 Jul;14(4):347-53.

18. Lucas, S. B., A. Hounnoun, C. Peacock, A. Beaumel, A. Kadio, and K. M. De Cock. 1994. Nocardiosis in HIV-positive patients: an autopsy study in West Africa. Tuber. Lung Dis. 75:301-307.

19. Reis, M. A., R. S. Costa, and A. S. Ferraz. 1995. Causes of death in renal transplant recipients: a study of 102 autopsies from 1968 to 1991. J. R. Soc. Med. 88:24-27.

20. McNeil, M. M., and J. M. Brown. 1994. The medically important aerobic actinomycetes: epidemiology and microbiology. Clin. Microbiol. Rev. 7:357-417.

21. Minero MV, Cercenado MME, Rabadan PM, Bouza E, Munoz P. 2009. Nocardiosis at the turn of the century.Medicine (Baltimore) 88:250 -261.

22. Ambrosioni J, Lew D, Garbino J. 2010. Nocardiosis: updated clinical review and experience at a tertiary center. Infection 38:89-97.

23. Gleadhill IC, Ferguson WP, Lowry RC. Efficacy and safety of ciprofloxacin in patients with respiratory infections in comparison with amoxycillin. Journal of Antimicrobial Chemotherapy. 1986 Nov 1;18(Supplement D):133-8.

24. Williams JH. Fluoroquinolones for respiratory infections: too valuable to overuse. Chest. 2001 Dec 1;120(6):1771-5.
25. Mamelak AN, Obana WG, Flaherty JF, et al. Nocardial brain abscess: treatment strategies and factors influencing outcome. Neurosurgery 1994; 35:622-631.

26. Zhao P, Zhang X, Du P, Li G, Li L, Li Z. Susceptibility profiles of Nocardia spp. to antimicrobial and antituberculotic agents detected by a microplateAlamar Blue assay. Scientific reports. 2017 Mar 2;7:43660.

27. Beaman, B. L. 1981. The possible role of Lphase variants of Nocardia in chronic infections. Zentbl. Bakteriol. Mikrobiol. Hyg. Abt. Suppl. 11:221- 227.

28. Beaman, B. L. 1982. Nocardiosis: role of the cell deficient state of Nocardia, p. 231-255. In G. J. Domingue (ed.), Cell wall defective bacteria: basic principles and clinical significance. Addison-Wesley Publishing Co., Inc., Reading, Mass.

29. Beaman, B. L. 1984. The cell wall as a determinant of pathogenicity in Nocardia: the role of L-forms in pathogenesis, p. 89-104. In L. Ortiz-Ortiz, L. F. Bojalil, and V. Yakoleff (ed.), Biological, biochemical and biomedical aspects of actinomycetes. Academic Press, Orlando, Fla.

30. Chapman, G., B. L. Beaman, D. A. Loeffler, D. M. Camp, E. F. Domino, D. W. Dickson, W. G. Ellis, I. Chen, S. E. Bachus, and P. A. Le Witt. 2003. In situ hybridization for detection of nocardial $16 S$ rRNA: reactivity within intracellular inclusions in experimentally infected cynomolgus monkeys and in Lewy body-containing human brain specimens. Exp. Neurol. 184:715-725.

31. Diaz-Corrales, F. J., C. Colasante, Q. Contreras, M. Puig, J. A. Serrano, L. Hernandez, and B. L. Beaman. 2004. Nocardia otitidiscaviarum (GAM-5) induces parkinsonian-like alterations in mouse. Braz. J. Med. Biol. Res. 37:539-548.

32. Riviere E, Neau D, Roux X, Lippa N, RogerSchmeltz J, Mercie P, Longy-Boursier M. Pulmonary streptomyces infection in patient with sarcoidosis, France, 2012. Emerging infectious diseases. 2012 Nov;18(11):1907. 